\title{
Focusing and Mode Separation of Elastic Vector Solitons in a 2D Soft Mechanical Metamaterial
}

\author{
Bolei Deng, ${ }^{1}$ Chengyang Mo, ${ }^{2}$ Vincent Tournat, ${ }^{3,1}$ Katia Bertoldi, ${ }^{1,4}$ and Jordan R. Raney," \\ ${ }^{1}$ Harvard John A. Paulson School of Engineering and Applied Sciences, Harvard University, Cambridge, Massachusetts 02138, USA \\ ${ }^{2}$ Department of Mechanical Engineering and Applied Mechanics, University of Pennsylvania, Philadelphia, Pennsylvania 19104, USA \\ ${ }^{3}$ Laboratoire d'Acoustique de l'Université du Mans, LAUM - UMR 6613 CNRS, Le Mans Université, France \\ ${ }^{4}$ Kavli Institute, Harvard University, Cambridge, Massachusetts 02138, USA
}

(Received 23 January 2019; published 10 July 2019)

\begin{abstract}
Soft mechanical metamaterials can support a rich set of dynamic responses, which, to date, have received relatively little attention. Here, we report experimental, numerical, and analytical results describing the behavior of an anisotropic two-dimensional flexible mechanical metamaterial when subjected to impact loading. We not only observe the propagation of elastic vector solitons with three components-two translational and one rotational-that are coupled together, but also very rich direction-dependent behaviors such as the formation of sound bullets and the separation of pulses into different solitary modes.
\end{abstract}

DOI: 10.1103/PhysRevLett.123.024101

Ongoing advances in digital manufacturing technologies are enabling fabrication of systems with an unprecedented level of compositional and structural complexity [1-3]. This remarkable control of geometry has stimulated major advances in the design of mechanical metamaterialsdesigner matter with unique mechanical properties that are dictated by their engineered structure [4,5]. While initial efforts in the field have focused on systems with unusual linear properties, such as negative Poisson's ratio [6-8], negative stiffness $[9,10]$, and negative thermal expansion $[11,12]$, large deformation and nonlinearities have been recently embraced as a means toward new functionalities, including programmability [13], energy absorption [14], and shape transformation [15]. Moreover, it has been shown that highly deformable mechanical metamaterials can be designed to support the propagation of a variety of nonlinear waves with large displacement amplitudes [16-19], providing a convenient platform to study nonlinear wave physics. However, to date the investigation of the nonlinear dynamic response of flexible metamaterials has been limited to one-dimensional (1D) systems.

Here, we investigate the nonlinear dynamic response of a 2D flexible mechanical metamaterial comprising a periodic arrangement of squares connected at their vertices by thin ligaments $[18,20,21]$. Remarkably, our experiments and analyses reveal that several new physical phenomena emerge when subjecting the structure to low-energy impacts. First, our system supports the propagation of elastic vector solitons with three polarization components- two translational and one rotational. Second, we investigate the effect of the anisotropy of the medium on the 2D nature of the soliton and find that such anisotropy plays a crucial role, leading to rich new nonlinear effects. For example, for propagation at $45^{\circ}$ from the symmetry axis, a distinct focusing effect is observed. The pulse does not spread along either direction, suggesting that sound bullets may exist in our system. Moreover, we find that for most other propagation angles the wave separates into two distinct solitary modes, each following a principal direction of symmetry. While 2D nonlinear elastic waves have been previously studied in granular media [22-25], the monolithicity and printability of our system allow facile control of the architecture, and hence control of the system's nonlinear dynamic response, providing a powerful platform to explore, visualize, and engineer new wave phenomena.

We start by studying experimentally the response of a 2D circular sample with 30 squares along its diameter when excited with an impactor [see Fig. 1(a)]. Our sample is fabricated out of polydimethylsiloxane using direct ink writing, an extrusion-based 3D printing approach [26]. Steel spheres with a diameter of $4.35 \mathrm{~mm}$ are embedded in the middle of the squares to modify their inertial properties. All squares are rotated by offset angles of $\theta_{0}=25^{\circ}$, have center-to-center-distance of $a=9.27 \mathrm{~mm}$, and are connected to one another by ligaments approximately $5 \mathrm{~mm}$ in width [see Fig. 1(b)]. In our experiments, we impact the sample at different points along its circumference to initiate pulses that propagate along different directions defined as

$$
\hat{\mathbf{e}}_{\|}=\cos \phi \hat{\mathbf{e}}_{x}+\sin \phi \hat{\mathbf{e}}_{y},
$$

where $\hat{\mathbf{e}}_{x}$ and $\hat{\mathbf{e}}_{y}$ denote the two directions of periodicity of the system, and impact angle $\phi$ is the angle between the normal to the impactor and $\hat{\mathbf{e}}_{x}$ [see Fig. 1(a)]. Finally, we record the impact event with a high speed camera, allowing measure of local vectorial displacement and velocity via digital image correlation [27,28] (see the Supplemental Material [29] for additional information). 

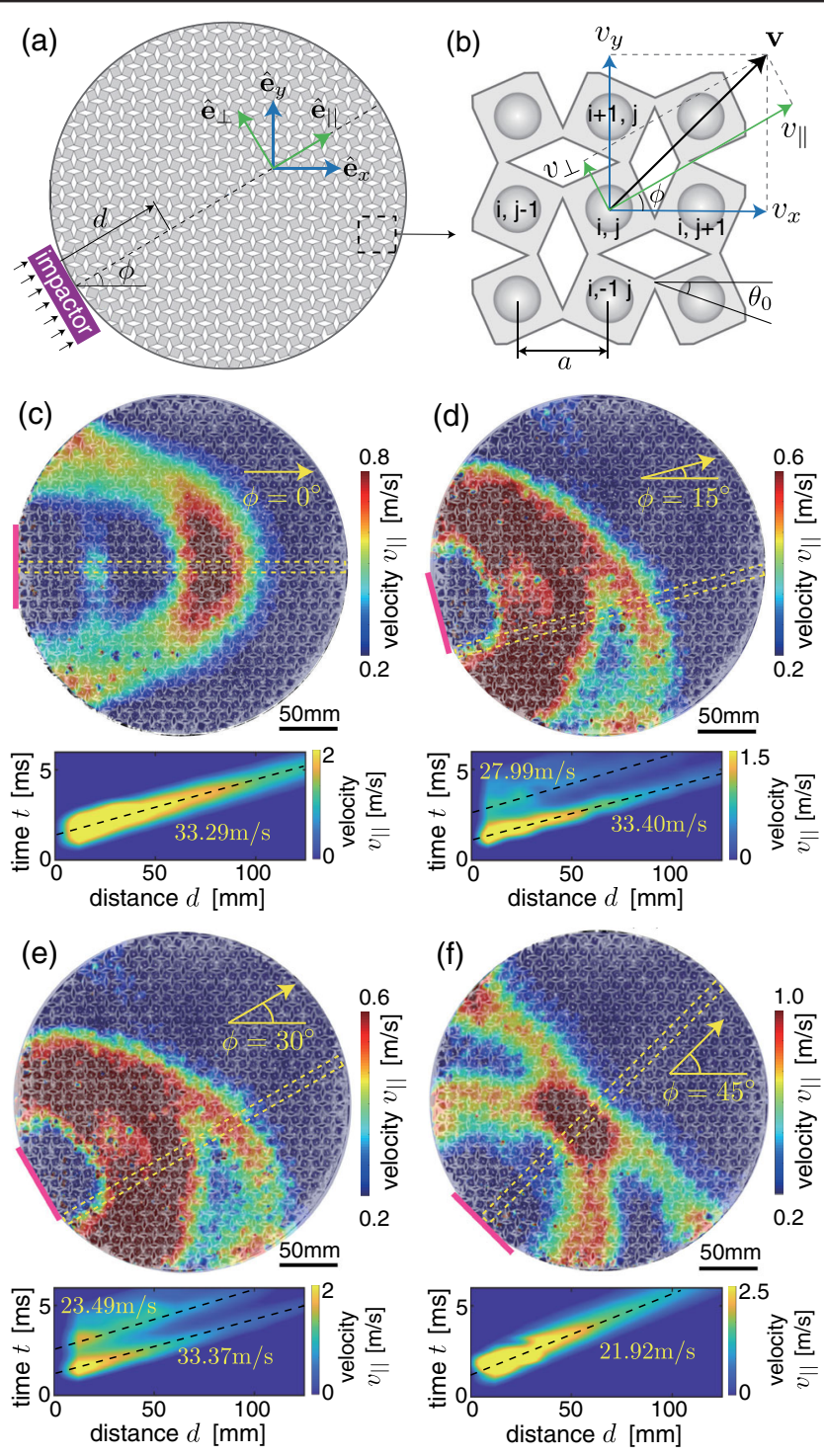

FIG. 1. (a) Schematic of the system. (b) Definition of velocity of squares. (c)-(f) Contour plots of parallel velocity $v_{\|}$and $t$ - $d$ contour plots of velocity along indicated direction for impact angles of $\phi=0^{\circ}, 15^{\circ}, 30^{\circ}$, and $45^{\circ}$. The magenta squares are those to which the displacement is applied. The full time evolution is available in Movie S1 of the Supplemental Material [29].

In Figs. 1(c)-1(f), we report contour plots of the velocity along $\hat{\mathbf{e}}_{\|}, v_{\|}$, at $t=5.5 \mathrm{~ms}$ after impact for $\phi=0^{\circ}, 15^{\circ}$, $30^{\circ}$, and $45^{\circ}$. Moreover, for each impact angle we also show the spatial-temporal evolution of $v_{\|}$along the propagation direction (focusing on the region delimited by the yellow dashed lines in the snapshots), allowing extraction of pulse speed (details in the Supplemental Material [29]). Three key features emerge from these plots. First, for impact angle $\phi=0^{\circ}$ the pulse propagates in a solitary fashion (i.e., it maintains both its shape and velocity), as the velocity profile is characterized by a single peak with nearly constant width. Second, for $\phi=15^{\circ}$ and $30^{\circ}$ the excitation

splits into two separate pulses. This is apparent both from the asymmetric velocity profile and from the two peaks seen in the spatial-temporal evolution of $v_{\|}$, each with constant velocity and pulse width. Third, for $\phi=45^{\circ}$ we again have a single pulse propagating through the sample, but this time the wave front keeps its shape in both the $\mathbf{e}_{\|}$and $\mathbf{e}_{\perp}$ directions. As a matter of fact, the pulse transversal width is the same as the impactor width (see the Supplemental Material [29] for more details). This suggests that for $\phi=45^{\circ}$ the wave has a transversal self-focusing effect, balancing the linear beam diffraction and stabilizing the pulse lateral width. This potentially leads to the generation of compact sound bullets of very large amplitudes, which may dramatically impact a variety of applications, such as biomedical devices, nondestructive evaluation, and defense systems [30-32].

To better understand our experimental results, we numerically model the system as an array of rigid squares connected at their vertices via a combination of linear axial (with stiffness $k_{s}=8180 \mathrm{~N} / \mathrm{m}$ and $k_{l}=16360 \mathrm{~N} / \mathrm{m}$ ) and rotational springs (with stiffness $k_{\theta}=0.0312 \mathrm{~N} \mathrm{~m} / \mathrm{rad}$ ) $[18,33,34]$. Moreover, we assign to the $[i, j]$ th square three degrees of freedom (DoF): the displacement in the $\hat{\mathbf{e}}_{x}$ direction, $u_{x}^{[i, j]}$, the displacement in the $\hat{\mathbf{e}}_{y}$ direction, $u_{y}^{[i, j]}$, and the rotation around the $z$ axis, $\theta^{[i, j]}$. Using these definitions, the equations of motion for the $[i, j]$ th square are given by

$$
m \ddot{u}_{\gamma}^{[i, j]}=\sum_{p=1}^{4} F_{p}^{\gamma[i, j]}, \quad J \ddot{\theta}^{[i, j]}=\sum_{p=1}^{4} M_{p}^{[i, j]},
$$

where $\gamma=x, y$, and $m=0.797 \mathrm{~g}$ and $J=5.457 \mathrm{~g} \mathrm{~mm}^{2}$ are, respectively, the mass and moment of inertia of the rigid units. Moreover, $F_{p}^{x[i, j]}$ and $F_{p}^{y[i, j]}$ are the forces along the $\hat{\mathbf{e}}_{x}$ and $\hat{\mathbf{e}}_{y}$ directions generated at the $p$ th vertex of the $[i, j]$ th unit by the springs, and $M_{p}^{[i, j]}$ represents the corresponding moment (see the Supplemental Material [29] for their explicit expressions).

By numerically solving Eq. (2) via the fourth order Runge-Kutta method, we find that the physical phenomena observed in our tests (i.e., solitonlike pulses, mode separation for $\phi=15^{\circ}$ and $30^{\circ}$, and self-focusing for $\phi=45^{\circ}$ ) not only persist, but actually become more accentuated when considering a larger model with 60 squares along the diameter (see Fig. 2 and the Supplemental Material [29]). Furthermore, in our numerical analysis we also excite planar waves on square-shaped samples and again observe solitonlike pulses and separation of modes (see Fig. S11 of the Supplemental Material [29]). As such, our numerical results indicate that the phenomena observed in the experiments are not artifacts introduced by either edge effects, damping or excitation, but rather emerge because of the bulk properties of the medium. 

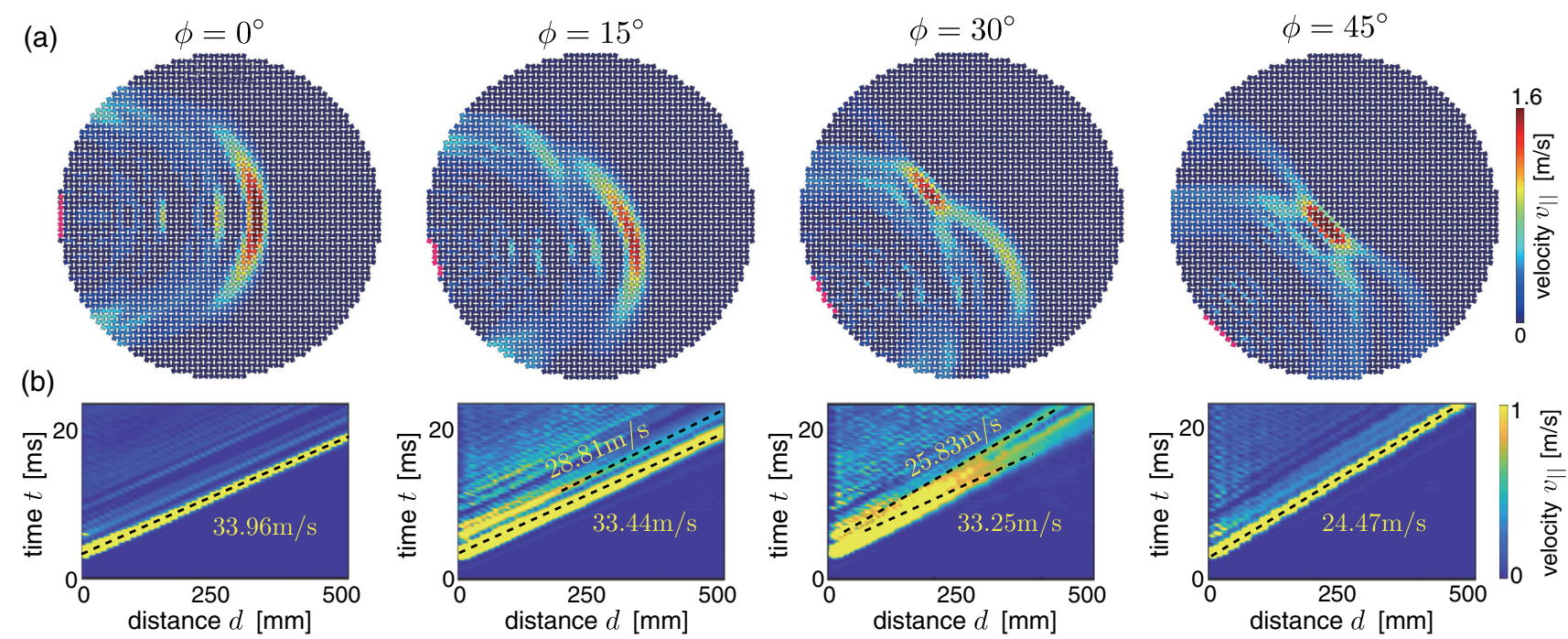

FIG. 2. Numerical results for a circular model with 60 squares along its diameter. (a) Contour plots of $v_{\|}$at $t=12 \mathrm{~ms}$ for all four impact angles. (b) Spatial-temporal map of $v_{\|}$for all considered impact angles. The magenta squares in (a) are those to which the displacement is applied. The full time evolution is available in Movie S2 of the Supplemental Material [29].
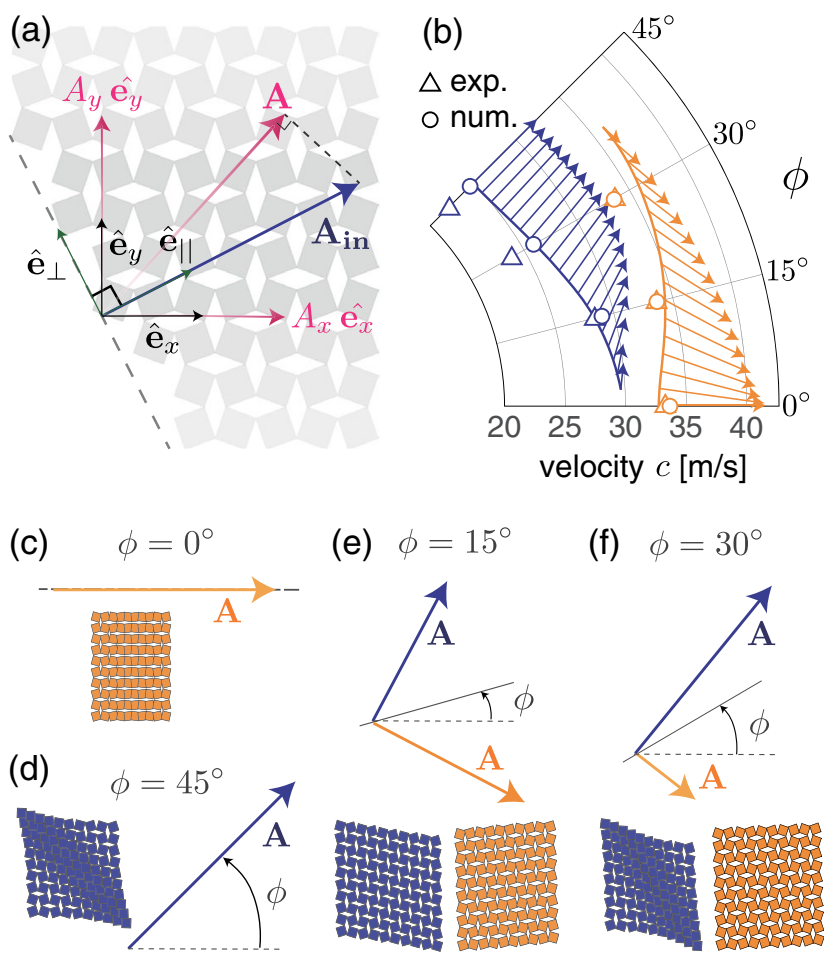

(f) $\phi=30^{\circ}$

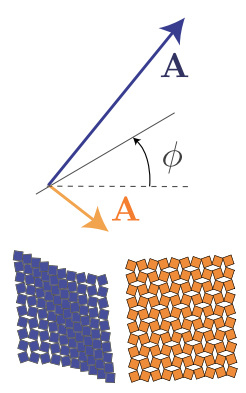

FIG. 3. (a) Schematic highlighting the input signal $\mathbf{A}_{\text {in }}$ and the translational amplitude of the excited soliton A. (b) Translational amplitude $\mathbf{A}$ of the solitons excited by impacts of amplitude $A_{\text {in }}=7 \mathrm{~mm}$ for $\phi \in\left[0,45^{\circ}\right]$. The triangular and circular markers correspond to the velocities extracted from our experimental and numerical results. (c)-(f) Translational amplitude and associated deformation for all solitary modes. Note that the displacements and rotations are five times amplified, excited by input signals with $A_{\text {in }}=7 \mathrm{~mm}$ and (c) $\phi=0^{\circ}$, (d) $\phi=45^{\circ}$, (e) $\phi=15^{\circ}$, and (f) $\phi=30^{\circ}$.
Since our numerical results reveal that the phenomena observed in our experiments are a robust feature of the system, we next explore deeper insights into the nonlinear dynamic properties of our system by simplifying Eq. (2) to derive analytical solutions for the case of planar waves. To this end, we assume that the wavelength of the propagating waves is much wider than the cell size $a$ and that $\theta^{[i, j]} \ll 1$. We then take the continuum limit of Eq. (2) and retain nonlinear terms up to the third order to obtain the continuum governing equations

$$
\begin{aligned}
m \ddot{u}_{x}= & a^{2}\left(k_{l} \partial_{x x} u_{x}+k_{s} \partial_{y y} u_{x}+\frac{\tan \theta_{0} a^{2} k_{l}}{6} \partial_{x x x} \theta\right. \\
& \left.+k_{l}\left(\tan \theta_{0}+\theta-\tan \theta_{0} \theta^{2} / 2\right) \partial_{x} \theta\right), \\
m \ddot{u_{y}}= & a^{2}\left[k_{s} \partial_{x x} u_{y}+k_{l} \partial_{y y} u_{y}+\frac{\tan \theta_{0} a^{2} k_{l}}{6} \partial_{y y y} \theta\right. \\
& \left.+k_{l}\left(\tan \theta_{0}+\theta-\tan \theta_{0} \theta^{2} / 2\right) \partial_{y} \theta\right],
\end{aligned}
$$

$$
\begin{aligned}
J \ddot{\theta}= & a^{2}\left(k_{s}-k_{l} \tan ^{2} \theta_{0}-4 k_{\theta}\right) \nabla^{2} \theta / 4 \\
& -2 a^{2}\left(k_{l} \tan ^{2} \theta_{0}+4 k_{\theta}\right) \theta-3 a^{2} k_{l} \tan \theta_{0} \theta^{2} \\
& -a^{2} k_{l}\left(\tan \theta_{0}+\theta-\tan \theta_{0} \theta^{2} / 2\right)\left(\partial_{x} u_{x}+\partial_{x} u_{y}\right) \\
& -a^{2} k_{l}\left(13-15 \tan ^{2} \theta_{0}\right) \theta^{3} / 12,
\end{aligned}
$$

where $\partial_{\gamma} f=\partial f / \partial \gamma, \nabla^{2}=\partial_{x x}+\partial_{y y}$, and $u_{x}, u_{y}$, and $\theta$ are three continuous functions which interpolate the discrete 
variables $u_{x}^{[i, j]}, u_{y}^{[i, j]}$, and $\theta^{[i, j]}$, respectively (see the Supplemental Material [29] for details).

To solve Eq. (3), we focus on planar waves propagating along the $\hat{\mathbf{e}}_{\|}$direction and introduce the traveling coordinate $\zeta=x \cos \phi+y \sin \phi-c t$, with $c$ being the pulse velocity. Introduction of $\zeta$ into Eq. (3), integration of Eqs. (3a) and (3b) with respect to $\zeta$ and their subsequent substitution into Eq. (3c), yields

$$
\mathrm{d}_{\zeta \zeta} \theta=C_{1} \theta+C_{2} \theta^{2}+C_{3} \theta^{3}
$$

with

$$
\begin{aligned}
& C_{1}=-4 F\left[\left(E_{1}^{x}+E_{1}^{y}-2\right) \sin ^{2} \theta_{0}-2 K_{\theta}\right], \\
& C_{2}=-3 F \sin 2 \theta_{0}\left(E_{1}^{x}+E_{1}^{y}-2\right), \\
& C_{3}=-F\left(7 \cos 2 \theta_{0}-1\right)\left(E_{1}^{x}+E_{1}^{y}-2\right) / 3,
\end{aligned}
$$

and

$$
\begin{aligned}
E_{\gamma}^{x} & =\frac{\cos ^{2 \alpha} \phi}{\cos ^{2} \phi+\frac{k_{s}}{k_{l}} \sin ^{2} \phi-\frac{m c^{2}}{k_{l} a^{2}}}, \\
E_{\gamma}^{y} & =\frac{\sin ^{2 \alpha} \phi}{\frac{k_{s}}{k_{l}} \cos ^{2} \phi+\sin ^{2} \phi-\frac{m c^{2}}{k_{l} a^{2}}}, \\
F & =\frac{3 k_{l} \sec ^{2} \theta_{0} / 2}{a^{2}\left[\frac{3 k_{s}}{2}+k_{l} \tan ^{2} \theta_{0}\left(E_{2}^{x}+E_{2}^{y}-\frac{3}{2}\right)\right]-6\left(k_{\theta}-\frac{k_{l} c^{2} J}{m a^{2}}\right)},
\end{aligned}
$$

where $\gamma=1,2$. Equation (4) can be directly derived from the Klein-Gordon equation with quadratic and cubic nonlinearities $[35,36]$, by substitution of the traveling wave coordinate $\zeta$. It admits well-known solitary wave solutions of the form

$$
\theta=\frac{1}{D_{1} \pm D_{2} \cosh (\zeta / W)},
$$

where

$D_{1}=-\frac{C_{2}}{3 C_{1}}, \quad D_{2}=\sqrt{\frac{C_{2}^{2}}{9 C_{1}^{2}}-\frac{C_{3}}{2 C_{1}}}, \quad$ and $\quad W=\frac{1}{\sqrt{C_{1}}}$.

Finally, the solution for the translational components $u_{x}$ and $u_{y}$ can be obtained by integrating Eqs. (3a) and (3b) with respect to $\zeta$ (see the Supplemental Material [29] for details).

Having obtained an analytical solitary wave solution, we now use it to validate our experimental and numerical observations. To begin with, we note that the analytical solution confirms that the pulses propagating in our $2 \mathrm{D}$ mechanical metamaterial are solitons. Specifically, it reveals that they are elastic vector solitons with three components - two translational and one rotational-that are coupled together and copropagate without dispersion. Note that although the springs used here are all linear, the nonlinearity still emerges from the " +1 " rotational DoF through its nonlinear geometrical coupling between the two translational DoF. Next, we use our continuum model to understand whether for specific loading directions $\phi$ the system supports solitary waves with different modes. To this end, we start by noting that in our experiments the impactor imposes a displacement with amplitude

$$
\mathbf{A}_{\text {in }}=A_{\text {in }} \hat{\mathbf{e}}_{\|}=A_{\text {in }} \cos \phi \hat{\mathbf{e}}_{x}+A_{\text {in }} \sin \phi \hat{\mathbf{e}}_{y},
$$

to the squares that it contacts. This input signal excites a vector soliton with translational amplitude

$$
\mathbf{A}=A_{x} \hat{\mathbf{e}}_{x}+A_{y} \hat{\mathbf{e}}_{y},
$$

where $A_{x}$ and $A_{y}$ are the amplitudes of its translational components, which are functions of both the propagation velocity $c$ and the propagation angle $\phi$ [i.e., $A_{x}(c, \phi)$ and $\left.A_{y}(c, \phi)\right]$ (see the Supplemental Material [29] for details). Since the translational amplitude should be a projection of the input signal along the direction of $\mathbf{A}$ [see Fig. 3(a)], it follows that

$$
|\mathbf{A}|=\sqrt{A_{x}^{2}+A_{y}^{2}}=\mathbf{A}_{\text {in }} \cdot \frac{\mathbf{A}}{|\mathbf{A}|},
$$

which provides a relation between the input signal applied by the impactor (i.e., the amplitude $A_{\text {in }}$ and the angle $\phi$ ) and the propagating velocity $c$ of the excited solitary wave. Therefore, given a pair of input parameters $\phi$ and $A_{\text {in }}$, Eq. (11) can be used to solve for $c$ and, once $c$ is known, the form of the solitary pulse excited by the impact via Eqs. (7), $(3 a)$, and (3b).

In Fig. 3(b), we show the evolution as a function of the angle $\phi$ of $\mathbf{A}$ and $c$ for solitons excited by impacts of amplitude $A_{\text {in }}=7 \mathrm{~mm}$ (the input displacement applied in our experiments). Interestingly, we find that for most impact directions two different solitary modes are excited by $\mathbf{A}_{\text {in }}$, each characterized by a distinct velocity $c$ and translational amplitude A. Only for impact directions of $\phi \sim 0^{\circ}$ and $\sim 45^{\circ}$ is a single wave mode excited. Importantly, we also find that the velocities predicted by our continuum model nicely agree with those extracted from both our experimental (triangular markers) and numerical (circular markers) results. Finally, to get a better understanding of the different solitary modes excited by the input signal, in Figs. 3(c)-3(f) we show a snapshot of the deformation induced by all excited modes for $\phi=0^{\circ}, 15^{\circ}, 30^{\circ}$, and $45^{\circ}$. We find that, while for $\phi=0^{\circ}$ and $45^{\circ}$ a pure compression wave propagates through the structure (i.e., all squares moves along the $\hat{\mathbf{e}}_{\|}$direction), for $\phi=15^{\circ}$ and $\phi=30^{\circ}$ two mixed compression-shear solitary modes are excited-a prediction that matches well with our experimental and numerical results.

Finally, we focus on the transversal self-focusing effect observed both in our experiments and numerical analyses 

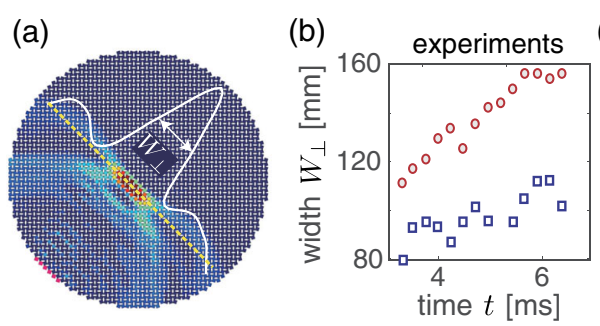

(c)

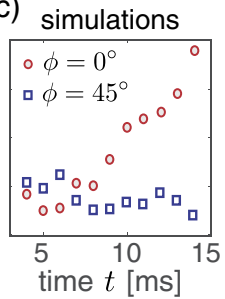

FIG. 4. (a) Schematic highlighting the full width at half maximum, $W_{\perp}$. (b),(c) Time evolution of $W_{\perp}$ as extracted from (b) experiments and (c) simulations for $\phi=0^{\circ}$ (red markers) and $\phi=45^{\circ}$ (blue markers).

for $\phi=45^{\circ}$. To better quantify it, for $\phi=0^{\circ}$ and $45^{\circ}$ we extract from both our experiments and numerical simulations the width of the propagating pulses along the $\hat{\mathbf{e}}_{\perp}$ direction, $W_{\perp}$ (see the Supplemental Material [29] for details). The results shown in Figs. 4(b) and 4(c) indicate that $W_{\perp}$ is rather constant with time (or equivalently distance) for $\phi=45^{\circ}$ and that its variation is significantly smaller relative to that observed for $\phi=0^{\circ}$. As such, this analysis confirms the self-focusing effect observed in the contour plots of Figs. 1 and 2 for $\phi=45^{\circ}$.

To summarize, we have used a combination of experimental, numerical, and analytical methods to study the propagation of nonlinear elastic waves in a $2 \mathrm{D}$ soft mechanical metamaterial comprising a network of squares connected by thin and highly deformable ligaments. Our results reveal that the system supports not only the propagation of elastic vector solitons with three components (two translational and one rotational), but also very rich behaviors such as compact pulses (akin to sound bullets) and separation of the pulses into different solitary modes. As such, our study shows that soft mechanical metamaterials provide a convenient platform to study nonlinear wave physics. Moreover, the 3D printability of these systems enables unique opportunities for engineering wave phenomena, ultimately providing new opportunities to control and manage intense vibrations and waves.

This research was partially supported by the National Science Foundation through the University of Pennsylvania Materials Research Science and Engineering Center (MRSEC) DMR-1720530.

B. D. and C. M. contributed equally to this work.

"Corresponding author. raney@seas.upenn.edu

[1] J. R. Raney and J. A. Lewis, Printing mesoscale architectures, MRS Bull. 40, 943 (2015).

[2] T. A. Schaedler and W.B. Carter, Architected cellular materials, Annu. Rev. Mater. Res. 46, 187 (2016).

[3] R. L. Truby and J. A. Lewis, Printing soft matter in three dimensions, Nature (London) 540, 371 (2016).
[4] J. Christensen, M. Kadic, O. Kraft, and M. Wegener, Vibrant times for mechanical metamaterials, MRS Commun. 5, 453 (2015).

[5] K. Bertoldi, V. Vitelli, J. Christensen, and M. van Hecke, Flexible mechanical metamaterials, Nat. Rev. 2, 17066 (2017).

[6] J. N. Grima and K. E. Evans, Auxetic behavior from rotating squares, J. Mater. Sci. Lett. 19, 1563 (2000).

[7] Y. Cho, J.-H. Shin, A. Costa, T. A. Kim, V. Kunin, J. Li, S. Yeon Lee, S. Yang, H. N. Han, I.-S. Choi, and D. J. Srolovitz, Engineering the shape and structure of materials by fractal cut, Proc. Natl. Acad. Sci. U.S.A. 111, 17390 (2014).

[8] A. Clausen, F. Wang, J. S. Jensen, O. Sigmund, and J. A. Lewis, Topology optimized architectures with programmable poisson's ratio over large deformations, Adv. Mater. 27, 5523 (2015).

[9] R. S. Lakes, T. Lee, A. Bersie, and Y. C. Wang, Extreme damping in composite materials with negative-stiffness inclusions, Nature (London) 410, 565 (2001).

[10] L. B. Kashdan, M. R. Haberman, P. S. Wilson, and C. C. Seepersad, Constrained negative stiffness metamaterials, J. Acoust. Soc. Am. 128, 2428 (2010).

[11] L. Wu, B. Li, and J. Zhou, Isotropic negative thermal expansion metamaterials, ACS Appl. Mater. Interfaces 8, 17721 (2016).

[12] Q. Wang, J. A. Jackson, Q. Ge, J. B. Hopkins, C. M. Spadaccini, and N.X. Fang, Lightweight Mechanical Metamaterials with Tunable Negative Thermal Expansion, Phys. Rev. Lett. 117, 175901 (2016).

[13] B. Florijn, C. Coulais, and M. van Hecke, Programmable Mechanical Metamaterials, Phys. Rev. Lett. 113, 175503 (2014).

[14] S. Shan, S. H. Kang, J. R. Raney, P. Wang, L. Fang, F. Candido, J. A. Lewis, and K. Bertoldi, Multistable architected materials for trapping elastic strain energy, Adv. Mater. 27, 4296 (2015).

[15] B. Haghpanah, L. Salari-Sharif, P. Pourrajab, J. Hopkins, and L. Valdevit, Multistable shape-reconfigurable architected materials, Adv. Mater. 28, 7915 (2016).

[16] J. R. Raney, N. Nadkarni, C. Daraio, D. M. Kochmann, J. A. Lewis, and K. Bertoldi, Stable propagation of mechanical signals in soft media using stored elastic energy, Proc. Natl. Acad. Sci. U.S.A. 113, 9722 (2016).

[17] N. Nadkarni, A. F. Arrieta, C. Chong, D. M. Kochmann, and C. Daraio, Unidirectional Transition Waves in Bistable Lattices, Phys. Rev. Lett. 116, 244501 (2016).

[18] B. Deng, J. R. Raney, V. Tournat, and K. Bertoldi, Elastic Vector Solitons in Soft Architected Materials, Phys. Rev. Lett. 118, 204102 (2017).

[19] H. Yasuda, Y. Miyazawa, E. G. Charalampidis, C. Chong, P. G. Kevrekidis, and J. Yang, Origami-based impact mitigation via rarefaction solitary wave creation, Sci. Adv. 5, eaau2835 (2019).

[20] M. Taylor, L. Francesconi, M. Gerendás, A. Shanian, C. Carson, and K. Bertoldi, Low porosity metallic periodic structures with negative poissons ratio, Adv. Mater. 26, 2365 (2014).

[21] C. Coulais, C. Kettenis, and M. van Hecke, A characteristic length scale causes anomalous size effects and boundary programmability in mechanical metamaterials, Nat. Phys. 14, 40 (2018). 
[22] R. K. Narisetti, M. Ruzzene, and M. J. Leamy, Study of wave propagation in strongly nonlinear periodic lattices using a harmonic balance approach, Wave Motion 49, 394 (2012).

[23] A. Leonard, C. Chong, P. G. Kevrekidis, and C. Daraio, Traveling waves in $2 \mathrm{~d}$ hexagonal granular crystal lattices, Granular Matter 16, 531 (2014).

[24] H. A. Burgoyne, J. A. Newman, W. C. Jackson, and C. Daraio, Guided impact mitigation in $2 \mathrm{~d}$ and $3 \mathrm{~d}$ granular crystals, Procedia Eng. 103, 52 (2015).

[25] S. Sen, T. R. Krishna Mohan, and M. Tiwari, Impact dispersion using 2D and 3D composite granular packing, KONA Powder Part. J. 34, 248 (2017).

[26] J. A. Lewis, Direct ink writing of $3 \mathrm{~d}$ functional materials, Adv. Funct. Mater. 16, 2193 (2006).

[27] T. C. Chu, W. F. Ranson, and M. A. Sutton, Applications of digital-image-correlation techniques to experimental mechanics, Exp. Mech. 25, 232 (1985).

[28] J. Blaber, B. Adair, and A. Antoniou, Ncorr: Open-source $2 \mathrm{~d}$ digital image correlation matlab software, Exp. Mech. 55, 1105 (2015).

[29] See Supplemental Material at http://link.aps.org/supplemental/ 10.1103/PhysRevLett.123.024101 for additional details about experimental, numerical, and analytical results.
[30] A. Spadoni and C. Daraio, Generation and control of sound bullets with a nonlinear acoustic lens, Proc. Natl. Acad. Sci. U.S.A. 107, 7230 (2010).

[31] C. M. Donahue, P. W. J. Anzel, L. Bonanomi, T. A. Keller, and C. Daraio, Experimental realization of a nonlinear acoustic lens with a tunable focus, Appl. Phys. Lett. 104, 014103 (2014).

[32] K. Li, P. Rizzo, and X. Ni, Alternative designs of acoustic lenses based on nonlinear solitary waves, J. Appl. Mech. 81, 071011 (2014).

[33] B. Deng, P. Wang, Q. He, V. Tournat, and K. Bertoldi, Metamaterials with amplitude gaps for elastic solitons, Nat. Commun. 9, 3410 (2018).

[34] B. Deng, V. Tournat, and K. Bertoldi, Effect of predeformation on the propagation of vector solitons in flexible mechanical metamaterials, Phys. Rev. E 98, 053001 (2018).

[35] A. Polyanin and V. Zaitsev, Handbook of Nonlinear Partial Differential Equations, 2nd ed. (Chapman and Hall/CRC, Boca Raton-London-New York, 2011).

[36] A.-M. Wazwaz, New solitons and kink solutions for the Gardner equation, Commun. Nonlinear Sci. Numer. Simul. 12, 1395 (2007). 